\title{
La Estrategia en las organizaciones y los limites desde la perspectiva social y humana
}

\section{Strategy in organizations and limits from the social and human perspective}

\author{
Diego Cardona-Arbeláez* \\ Institución Tecnológica Colegio Mayor de Bolívar - \\ Colombia \\ ORCID iD: https://orcid.org/0000-0002-9123-0156 \\ dcardona@colmayorbolivar.edu.co \\ Natalia Gómez-Castillo \\ Universidad Tecnológica De Bolívar - Colombia \\ ncastillo@utb.edu.co
}

\section{Susana Margarita Lorduy-Miranda}

Universidad Tecnológica De Bolívar - Colombia

slorduy@utb.edu.co

* Autor a quien debe ser dirigida la correspondencia

\author{
Fecha de recepción: 02/11/2019 \\ Fecha de evaluación: 05/01/2020 \\ Fecha de aceptación: 03/05/2020
}

Cómo citar: Cardona-Arbeláez, D., Gómez-Castillo, N., \& Lorduy-Miranda, S. (2020). La Estrategia en las organizaciones y los limites desde la perspectiva social y humana. Revista Cientifica Anfibios, 3(1), 19-27. https://doi.org/10.37979/afb.2020v3n1.58

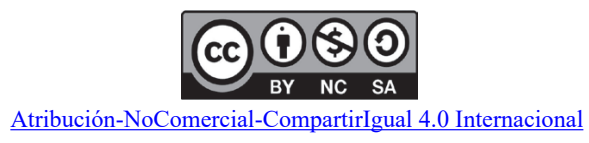

\section{Resumen}

El concepto de estrategia es ampliamente abordado en la literatura relacionada con la gestión organizacional, desde la primeras definiciones hasta las definiciones modernas le han aportado a la contrucción de organizaciones con una visión más amplia, enfocadas a la consecución de metas mas ambiciosas. El presente documento tiene por objetivo abordar las diferentes acesiones del concepto estrategia desde la perpectiva de diversos eruditos de este area de conocimiento haciendo gran enfasis en el modelo propuesto por Renée Bedard. Se puede concluir que el concepto estrategia es el lemento dinamizador y estructurador de las empresas, puesto que este posee la capcidad de dar norte a las acciones desarrolladas por el capital humano dentro de las organizaciones.

\section{Palabras Clave}

Estrategia; Enfoques; Líderes; Organización; Rombo de Renée Bedard

\begin{abstract}
The concept of strategy is extensively addressed in the literature related to organizational management, from the first definitions to modern definitions have contributed to the construction of organizations with a broader vision, focused on the achievement of more ambitious goals. This document aims to address the different meanings of the concept of strategy from the perspective of various scholars in this area of knowledge, placing great emphasis on the model proposed by Renée Bedard. It can be concluded that the concept of strategy is the dynamic and structuring element of companies, since it has the capacity to give direction to the actions developed by human capital within organizations.
\end{abstract}

\section{Keywords}

Strategy; Approaches; Leaders; Organization; Renée Bedard's Rhombus 


\section{Introducción}

Estrategia es una palabra con la que intuitivamente todo adulto está enormemente familiarizado, e incluso se podría llegar a decir que una persona promedio al ser confrontada con la pregunta, ¿Qué es estrategia?, ofrecería una aproximación a una definición, seguro de que está al menos abordando el tema desde una posición bastante alejada de la ignorancia. Seguramente no todos ofrecerán la misma respuesta, pero todos giran alrededor de algo similar a un plan. Ante un cuestionamiento común, esa respuesta común es suficiente, y satisface la mente de la gran mayoría. Para el común denominador, la estrategia es un plan, una forma de hacer las cosas.

Se puede ahondar y preguntar de vuelta, ¿y para qué se usa la estrategia?, y ahí uno puede encontrar mayor variedad de respuestas, pero eventualmente lo más básico resulta ser el concepto de salir victorioso de algo. Esa manera intuitiva de ligar estrategia con los conceptos de competencia y victoria es la que seguramente despertó algo en todo aquel que ha pretendido ahondar en el tema y aplicarlo al mundo que nos rodea. En la antigüedad la estrategia tenía un tono militar, ya que la mayoría de las contiendas eran bélicas y se luchaban en la arena a sangre y fuego. Vencer era vencer al enemigo, usando ejércitos como peones y alfiles en un tablero de ajedrez de tierra. En los siglos XX y XXI, aunque la guerra siguió siendo desgraciadamente una gran parte de la realidad y la erradicación humana se volvió tan fácil como oprimir un botón, las grandes mentes giraron su atención a otra arena, menos sangrienta pero no menos contenciosa. La estrategia dejó de ser propiedad únicamente de líderes como Sun Tzu y Napoleón, y emergieron grandes pensadores como Porter, Chandler, Miles, Snow, Ansoff, Kotler y Mintzberg. Su atención se volcó hacia la arena de los negocios, donde los grandes capitales luchan por triunfar día tras día, por acumular más riqueza y asegurar que perdure. Los titanes cambiaron la armadura de metal por un traje de saco y corbata, la espada por el plumero, y por ende el estudio de la estrategia desde lo académico gira hacia el análisis de la estrategia en un contexto empresarial.

\section{La estrategia}

La estrategia en el mundo empresarial tiene muchos matices y no se reduce a una única corriente de pensamiento. Es por ello que existe un abanico de expertos que presentan visiones ligeramente distintas, todas partiendo de lo intuitivo y conscientes de lo que se ha propuesto antes, pero con ánimos de ofrecer la visión más completa y que resulte más útil de estudiar y aplicar.

Partiendo con Porter, "La estrategia competitiva consiste en ser diferente. Significa la selección deliberada de un conjunto de actividades distintas para entregar una mezcla única de valor". Dicho de otra forma, "En un nivel fundamental, la estrategia para Porter se reduce en dos opciones muy amplias: Hacer lo que todos hacen (pero gastar menos plata haciéndolo), o hacer algo que nadie más puede hacer" (Ovans, 2015). Esta visión establece un vínculo directo entre la estrategia y el entorno donde se ubica el negocio, donde está esperando la competencia, posiblemente armada hasta los dientes. "Una estrategia competitiva explica cómo vas a hacerlo mejor que tus rivales, y hacerlo mejor, por definición, significa ser diferente" (Magretta, 2002). Se puede apreciar la hostilidad y la agresividad en estas definiciones, que aunque no implican derramamiento de sangre, sí traen a colación la competencia desenfrenada, la necesidad de competir para derrotar al enemigo y sólo a través de eso poder seguir vivo. Esto no sugiere una violencia de parte de Porter, sino del mundo empresarial como tal.

En su estudio de la estrategia, Porter se destaca por su concepto de las Cinco Fuerzas, donde afirma que "la competencia por las utilidades va más allá de los rivales establecidos de un sector e incluye a cuatro otras fuerzas competitivas: los clientes, los proveedores, los posibles entrantes y los productos sustitutos" (Porter, 2008). Enfocado en la industria, Porter pone la lupa sobre los factores externos a la empresa y la rivalidad propia de la industria. Estudia el poder de negociación de clientes y proveedores y cómo el aumento de poder de ellos es inversamente proporcional al de la empresa. Estudia la amenaza de nuevos competidores y la importancia de crear barreras de entrada, evocando memorias de los campos minados de guerras pasadas. Por último, 
recoge la amenaza de la aparición de productos sustitutos, que entrarían a afectar negativamente la rentabilidad del sector. Todo este conocimiento, de conocer al enemigo, de conocer el terreno, y de conocerse a sí mismo, está orientado a preparar a la empresa para sobrevivir y crecer. Su postulación de las Cinco Fuerzas revolucionó el mundo de los negocios, y sigue siendo el canon obligado para todos aquellos que se imaginan como futuros titanes de industria.

Posteriormente, y preocupado por la visión mercenaria que se tiene de la industria en general, Porter complementa su teoría de negocios al abordar el concepto de valor compartido, donde "El concepto de valor compartido puede ser definido como las políticas y las prácticas operacionales que mejoran la competitividad de una empresa a la vez que ayudan a mejorar las condiciones económicas y sociales en las comunidades donde opera" (Kramer, Porter, 2011). La idea principal sigue estando basada en generar utilidades, en aumentar la riqueza, pero le da un giro al atar la estrategia empresarial con la social, donde sus destinos están ligados y el éxito de una implica y necesita del éxito de la otra. Aunque el triunfo empresarial beneficia a todos cuyo ambiente ocupa esa empresa, sigue siendo necesario para esa empresa salir triunfante de la arena frente a sus competidores. De lo contrario, no le sirve a nadie.

Una concepción diferente de estrategia es la que ofrecen Miles \& Snow, que parten de la hipótesis que "las organizaciones con éxito desarrollan a lo largo del tiempo una adaptación sistemática al entorno" (Carmona, 2018), lo que llaman el ciclo adaptativo. Miles \& Snow resumen los problemas que pueden enfrentar las empresas en tres categorías, los de producto/mercado o de tipo negocio en el que operan, los de ingeniería o tipo técnicos o tecnológicos, y los de tipo administrativo que tienen que ver con la estructura de la empresa. Adicionalmente, postulan que existen básicamente cuatro orientaciones o posiciones estratégicas que asumen las empresas en la realidad. La primera es la Prospectiva, donde la empresa tiende a ser la punta de lanza en desarrollos de nuevos productos y está constantemente analizando el mercado para responder con prontitud. La segunda es Defensiva donde a diferencia de la anterior, las empresas se preocupan por el nicho de mercado que ocupan y ofrecen un portafolio de productos menos cambiante. La tercera es la Analizadora, que combina de cierto modo las dos anteriores, ya que aunque presenta estabilidad en sus productos y mercados, a lo defensivo, también está en búsqueda de nuevos mercados y oportunidades, según se vaya desarrollando la situación interna y externa. Por último identifican la posición Reactiva, que carece de estrategia y por lo tanto es inestable (Carmona, 2018).

La propuesta de Miles \& Snow busca entender las empresas, busca explicar la realidad que enfrentan y las posturas que asumen, para que a través de ese análisis introspectivo sobre el comportamiento interno y externo de una empresa, se logre un mejor rendimiento económico, es decir, mayor utilidad. Es claro que destacan y valoran una estrategia que esté atenta a las señales que da el entorno, que valoran la búsqueda y aprovechamiento de nuevas oportunidades al saber leer las situaciones que se desarrollan en su entorno, y que consideran como el nivel más bajo un posicionamiento estratégico reactivo que carece de fundamento, de planeación y de convicción.

Partiendo de Porter, Miles \& Snow, resulta útil el planteamiento planteado por Mintzberg, que trata de cierta forma de complementar la visión excesivamente externa de Porter. Su planteamiento de los posibles tipos de estrategia que puede adoptar una empresa es más extensa y específica que la de Porter, Miles y Snow, de cierta forma parece usarlos como punto de partida. De acuerdo a su planteamiento, las estrategias de diferenciación pueden ser por imagen, calidad, diseño, precio y soporte. Quizás lo más revolucionario es el concepto de estrategia de indiferenciación, que se refiere a aquellas empresas que se dedican a la imitación o que simplemente no tienen un factor diferenciador (Carmona, 2018). Para Porter, que insiste en ser mejor siendo diferente, una estrategia de ese tipo es blasfemia y propia de empresas que servirán de comida para otras. Es interesante que Mintzberg les proporcione su propia categoría a estas empresas, que valga decir, abundan a nivel global, y que se esfuerzan por sobrevivir de un día a otro. La impresión que deja el espíritu competitivo de la postulación de Porter es que fuera de merecer una categoría propia y ser reconocidas, podrían 
ser ignoradas por estar ya camino al cementerio. Sirven como lección y para ser absorbidas, ya que competir sin estrategia es casi que competir con una espada de madera y con miopía.

El prisma a través del cual Kotler analiza la estrategia es la participación de mercado, una perspectiva que mira hacia afuera al igual que lo hace Porter. Partiendo de ahí, identifica la estrategia líder, del retador, del seguidor y del especialista (Carmona, 2018). El líder es aquella empresa que cuenta con una posición dominante en el mercado, y que como cualquier Rey medieval, debe permanecer en la defensa constante de sus fronteras y de su posición. Siempre son un referente y están obligados a vivir en estado de inconformismo y tienen prohibido descansar sobre sus laureles, so pena de muerte. La estrategia del retador es propia de aquellas empresas que ocupan un alto porcentaje del mercado y están en una lucha por llegar a una posición dominante, buscando desplazar así al líder actual. ¡Muerto el Rey, viva el Rey! La estrategia del seguidor es afín de las empresas que no buscan tomarse La Corona en el campo de batalla, y que prefieren existir sin mayores sobresaltos $\mathrm{y}$ aspiraciones de grandeza. Por último destaca a la estrategia especialista, típica de empresas que buscan especializarse en un segmento de mercado muy pequeño, un nicho. Ese se vuelve su feudo y debe poder sostener a la empresa con lo que hay dentro de sus fronteras, y será defendido por ese pequeño ejército.

Quizás de las postulaciones que más importancia dan al concepto de estrategia es la de Chandler, que la ubica como la piedra sobre la cual construye su iglesia, o más específicamente, estableciendo a la estrategia como el componente primario que fija los criterios para luego escoger entre las distintas formas organizacionales, con sus ventajas y desventajas. Con eso en mente, se fija la estructura y se asigna la autoridad y el poder dentro de la organización, y de ahí sigue a procesos, recompensas y gente (Corporate Executive Board, 2009). De esta forma se prioriza que todo el diseño organizacional sea compatible con la estrategia y el modelo de negocio. Chandler postuló que la estructura sigue a la estrategia. Es decir, que todos los aspectos de la estructura de una organización se deben diseñar con base en la estrategia definida, y de esta forma se organiza a la empresa para optimizar el logro de objetivos estratégicos (Rhodes, 2011). De esta forma, se plantea que "el objetivo fundamental del establecimiento de la estructura primaria es dar respuesta organizativa a la estrategia corporativa elegida por la empresa" (Claver, Pertusa \& Molina, 2004, 2005). De esta forma le lleva incluso la contraria a Adam Smith, volviendo a la gerencia estratégica una mano visible que dirige la empresa y forja su destino a partir de la estrategia, desconociendo la idea de que el mercado se ajustaría con aquella mano invisible de otrora.

Las diferentes visiones de estrategia buscan todas lo mismo, y es la maximización de utilidades a través de una planeación, un posicionamiento y una acción premeditada e intencionada. Distintas escuelas perciben y destacan distintos atributos o características, pero la realidad es que muchas son complementarias y solamente explican otra dimensión del mismo problema. Al final del día, a todos les preocupa lo mismo, entender lo que ocurre y por qué, poder explicarlo a través del prisma de su lógica y conocimiento, y poder enseñarlo para que otros logren aprovechar ese conocimiento a su favor. Estrategia implica conocerse más y conocer más el entorno y tomar decisiones de acorde con esa realidad, y eso conlleva estar mejor preparado para esa guerra de negocios que los envuelve.

\section{Análisis desde un enfoque crítico de una estrategia basada en una guerra disfrazada}

Comprender la estrategia de una organización es saber cómo actúa ante diferentes eventualidades, como alcanza sus objetivos y cómo se desarrolla su estructura corporativa, basado en una Guerra de Saco y Corbata y un nuevo paradigma que se ha levantado en esta sociedad postmoderna que es "Hay que Engañar para Triunfar". Es entonces, donde basados en la estrategia de las Cinco fuerzas de Porter, explicado anteriormente, miraremos los postulados y contextualización de la aplicación del Rombo de Renné Bedard a una organización y esbozaremos una propuesta a la pregunta ¿Cómo cambiar una cultura organizacional arraigada al individualismo, hedonismo y racionalismo en una empresa ventanera de la ciudad de Barranquilla?

En contextualización, esta empresa ventanera se ha abierto camino en medio de grandes corporaciones nacionales e internacionales y a lo largo del tiempo ha desarrollado diferentes métodos de 
enseñanza y transferencia de información interna, buscando cambiar una cultura tóxica, basada en el conocimiento absoluto y la falta de compromiso colaborativo al ignorar los errores, buscando un beneficio propio.

El Rombo de Bédard también se puede comprar con la metáfora del Iceberg Organizacional en la que se expone los componentes visibles como la punta del iceberg, las operaciones y tareas diarias, conocimiento y habilidades, al aplicarlo a las organizaciones se vería reflejado en los métodos, metodologías, procedimientos, políticas, objetivos, entre otras y el hielo en profundidad, son el componente afectivo- emocionales orientados hacia lo socio-psicológico, es decir, los valores, los rasgos de las persona, normas grupales, pecepciones y actitudes de las personas.

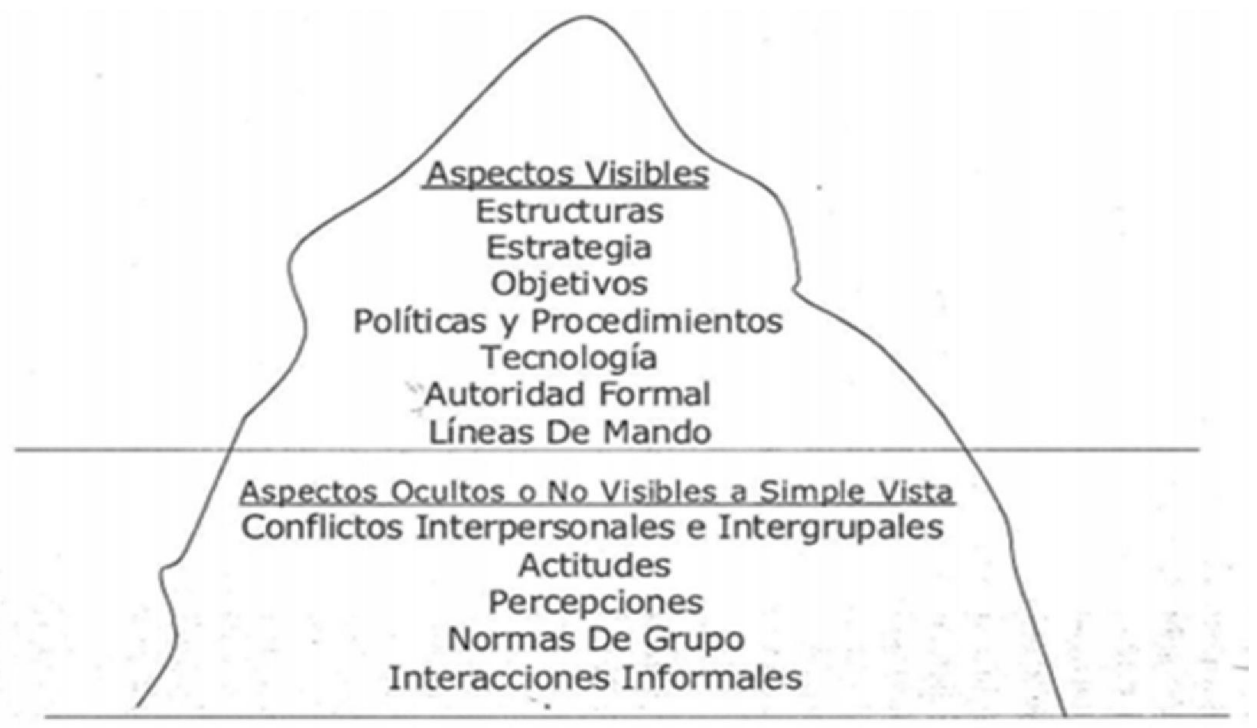

Figura 1. Metáfora de la Organización como Iceberg. Fuente: Aira (2016).

Al engranar esta metáfora con el Rombo de Bedard, se realiza simbiosis de estos modelos, con respecto a estas cuatro dimensiones filosóficas, entonces, la parte visible sería la Praxiología, es decir, las acciones, costumbres, el hacer de los colaboradores, pero todo tiene como base las experiencias, valores, conocimiento y se explican a través de las otras dimensiones como lo muestra la Figura 2.

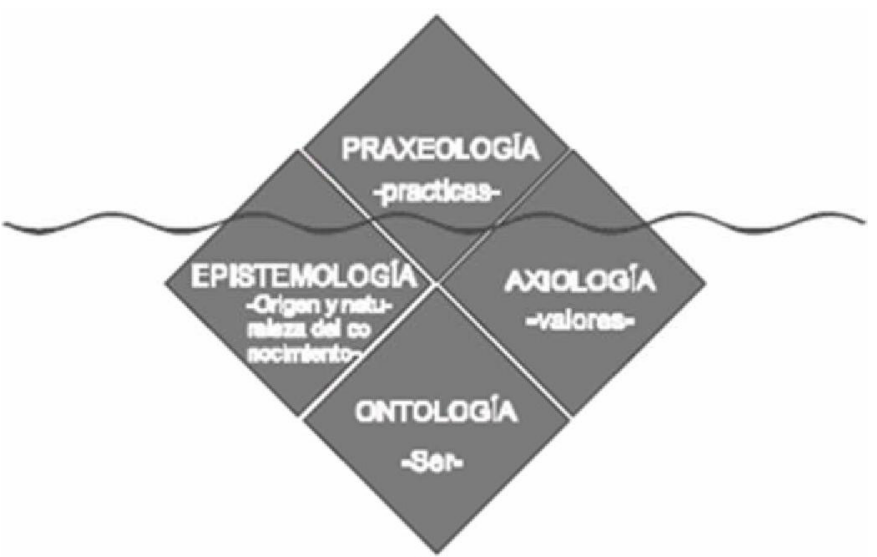

Figura 2 Rombo Filosófico de Renée Bedard

Fuente: Dávila, 2003. Extraído de González, C; Saltaren, H; Arenas, N. (2010) 
Cuando una organización busca cumplir los objetivos de maximizar sus ganancias, muchas veces, entran en declive los componentes que sostienen la punta del Iceberg, sobre todo si esta organización se mueve en un océano rojo, con productos sustitutos y amenazas de nuevos competidores. Pero, ¿por qué afirmar que entran en declive algunos componentes?, partiendo de la premisa que comprende el concepto de Estructura o Diseño organizacional como lo define Henry Mintzberg (1984) "Una organización efectiva es aquella que logra coherencia entre sus componentes y que no cambia un elemento sin evaluar las consecuencias en los otros", es decir que la estructura permite o dificulta el logro de las estrategias planteadas por las empresas y en esta caso nos referimos a los colaboradores como la estructura.

Jim Collins en el 2001, explica en su libro "Empresas que Sobresalen. Por qué unas sí pueden mejorar la rentabilidad y otras no", que ejecutivos iniciaron las transformaciones de una empresa buena a una sobresaliente, seleccionando primeramente personas competentes, y al colocarlas en los puestos adecuados, podrán definir entonces a qué lugar se dirigen. Sabiendo de antemano que cuenta con personal idóneo y calificado, capaz de ser preciso y riguroso. Ahora, realizaremos un análisis crítico de cómo se evidencia y aplica el rombo en esta empresa Ventaneria de la ciudad de Barranquilla.

\section{La competencia bajo los tres conceptos que componen la praxiología}

Para entender la praxiología en una organización, es importante resaltar tres conceptos, la Metodología, la Logia y la Praxis. Al interrelacionar estos conceptos, nos da como resultado que la praxeología es la metodología que busca estudiar la lógica del comportamiento Humano-Organizacional, como anteriormente se mencionó, es la punta del Iceberg, y no todo lo que se ve a simple vista es lo que motiva a las personas en su actuar. Bedard manifiesta que las competencias de una persona se definen por dos constituyentes del ser: El conocimiento que se comparte entre colaboradores afines y/o generales y el YO de cada individuo, es decir, la estructura propia con la que cuentan. Entonces, en la Praxiología en- contramos las prácticas y las conductas que buscan una mejora, pero cómo hacer que esta no se torne individualista y alejada del cooperativismo. Planteado una estrategia de retroalimentación y evaluación del comportamiento, para luego tomar un plan de acción de mejora y proseguir con una evolución creativa.

\section{Los valores como cuerpo del iceberg}

En una organización, es de vital importancia que su estructura tenga buenos cimientos, capaces de sostener el peso de la competitividad, sin caer en las tramas, engaños y la confusión a los clientes, por ello se evidencia en muchos casos que las empresas colocan medianos periodos de prueba, para saber si sus colaboradores cuentan con lo necesario para pertenecer a la institución, es donde, cobra importancia la axiología, que se puede definir como la teoría de la ciencia de los valores, justificada y legitimada en los valores sociales, culturales e individuales de cada ser, a su vez, estos se fundamentan en la ética y la moral. El problema que se presentan en esta organización, está basado en las crisis de la orientación de los valores corporativos, debido a que cada colaborador está buscando fines lucrativos individualistas, con base en el racionalismo, el individualismo -ya antes nombrado- y el hedonismo, y no moralmente aceptables bajo el concepto de la ética que la expondremos, como el conjunto de normas que justifican la conducta de un individuo dentro de una sociedad, entonces la organización se ve afectada en la búsqueda de los objetivos, por que presenta conflictos internos y estos se ven reflejado poco a poco en la Praxis.

Como atacar esta problemática actual, consideramos una reevaluación e incluir más valores corporativos, para permitir un crearán un nuevo perfil axiológico de la sociedad en la que se desenvuelve la empresa y aumenta el índice del desarrollo humano para resolver problemas de forma individual y social, por lo tanto las organizaciones deben actuar con madurez y sensibilidad social por su entorno inmediato y la sociedad en general. (Navas y González, 2016)

\section{El conocimiento como objeto de estudio}

Otra base del cuerpo de una organización que a simple vista no es evidenciable, es la episte- 
mología, citaremos en esta ocasión una de las frases célebres del Libertador Simón Bolívar que afirmaba y cuya credibilidad se ha corroborado es, "Un pueblo ignorante es un instrumento ciego de su propia destrucción", sin el conocimiento o fundamento teórico, no es posible dar forma a la práctica, que se verá reflejado en un Iceberg completamente sostenible y con larga durabilidad. Sin el componente del conocimiento, es muy difícil encontrar un hilo conductor que enlace la funcionalidad con la maximización de las ganancias operativas, pues este componente da seguridad en los procesos organizativos, ratifica hechos (utilidad) a través de los indicadores, rectifica el posicionamiento en que se encuentra la empresa y afirma las verdades expositivas ante perfiles competitivos que le permiten tener un factor diferenciador en la guerra vivencial de ser el mejor en el mercado. Pero todos estos factores no son posible sin que los colaboradores no busquen un conocimiento actualizado, con una constituyente histórica, pues se puede decir que la epistemología supervisa y controla las actividades de lógica del comportamiento Humano-Organizacional, pues este da soporte al desempeño. En este caso planteamos unos puentes que se desarrollen y ejecuten programas de investigación de diferentes estudios realizados internamente y que tengan un integrante dinámico del conocimiento, la tecnología, la innovación y la ciencia, se implementará grupos en coordinación con las universidades para mantener actualizado a nuestro Desarrollo Humano.

\section{El soporte de una estructura rígida y duradera}

La Ontología le da un significado al concepto de desempeño. En el artículo los fundamentos del pensamiento y las prácticas administrativas (2003), Renée Bédard afirma que la ontología suministra las grandes ideas directrices que permiten a cada uno orientarse, asegura la inteligibilidad de base y procura las certidumbres fundamentales; ella ofrece una cierta lectura del universo, una primera concepción de la realidad. En la organización debemos velar por imitar aquellas acciones correctas y fundamentadas epistemológica y axiológicamente para dar sentido a la praxiología del desempeño organizacional, buscando siempre pensar diferente, es decir innovar, en medio de una cultura diferente rodeándonos de un equipo de trabajo complementario y con las mismas bases ético-morales que hagan de un problema una oportunidad de mejora, para llevar a la organización al éxito sostenido.

\section{Conclusiones}

En una organización que se evidencia que sin una unificación de los valores, el arraigo de estos, la desestimación del individualismo, el hedonismo y el racionalismo, sin el fundamento epistémico que lleve a la búsqueda constante de las mejoras basados en la innovación, la trascendencia de una realidad permanente y estable, no es posible asegurar una trazabilidad en un océano rojo, entonces se deberá recurrir a muchas estrategias desgastantes para mantener a flote una empresa, cuyos colaboradores la pueden estar destruyendo desde su núcleo. Por ello se plantea, basado en la corriente filosófica del Rombo de Bédard cuatro espacios de desempeño corporativo: Área Informativa, Área de Comunicación, Área de Colaboración y el Área de Reflexión.

Lo que a largo plazo se busca es evidenciar que existen brechas entre el conocimiento adquirido con la formación y el conocimiento que realmente se necesita, además que la transferencia de conocimiento asegura la trazabilidad de los procesos y el individualismo afecta el éxito que una organización busca, alternado la tan esperada maximización de las ganancias, para cambiar el pensamiento es necesario dejar de pensar el yo para pensar en el nosotros y con el nosotros se verá que la mejora continua ante cualquier vicisitud o avatar en el mercado de desarrollo.

\section{Referencias}

Aira, M. R. (2016). Las instituciones y su incidencia en el comportamiento organizacional. Negotium: revista de ciencias gerenciales, 11(33), 99-111.

Balza-Franco, V., Paternina-Arboleda, C., \& Cardona-Arbeláez, D. (2019). Prácticas Colaborativas en la Cadena de Suministro: Una Revisión Conceptual. Saber, Ciencia y Libertad, 14(2), 77-101. 
Carmona-De Ríos, C. (2018). ¿Cuál es la importancia de implementar estrategias en las organizaciones?. Revista Científica Anfibios, 1(1), 71-79. https://doi.org/10.37979/afb.2018v1n1.20

Claver, Enrique; Pertusa, Eva María; Molina, José F. Configuraciones organizativas: congruencia entre el entorno, la estrategia y la estructura. Revista de Economía y Empresa, $N^{\circ} 52$ y 53 (2 Época) $3^{\circ}$ Cuatrimestre 2004 y $1^{\circ}$ Cuatrimestre 2005, pp. 197-211, Universidad de Alicante, España.

Corporate Executive Board, (2009). Frameworks for Organizational Design. Executive Brief. CLC6422289.

Gómez, Jorge. (Febrero 2 de 2017). ¿Qué es primero. La estrategia o El estratega?. Revista Dinero. https://www.dinero.com/opinion/columnistas/articulo/que-es-primero-la-estrategiao-el-estratega-por-jorge-gomez/241665.

Campo, C. H. G., Quiñónez, H. A. S., \& Quiñones, N. M. A. (2010). Modelo explicativo del conocimiento organizacional en la Corporación Autónoma Regional del Valle del Cauca-CVC. Revista Venezolana de Gerencia, 15(51), 388-406.

Kramer, Mark R. Porter, Michael E., (2011). La creación del Valor Compartido. Harvard Business Review América Latina, Enero-Febrero 2011, USA.

Magretta, Joan, (2002). Why Business Models Matter. Harvard Business Review, May 2002, R0205F, USA.

Mintzberg, Henry., Quinn, J., \& Voyer, J. (1997). El proceso estratégico: conceptos, contextos y casos. México: Pearson Education.

Miles, Raymond E., Charles C. Snow. Organizational Strategy, Structure, and Process. New York: McGraw-Hill, 1978.

Montenegro- Velandia, Wilson et al. (2016). Fundamentos epistemológicos que se articulan en la enseñanza de las temáticas Estructura - Estrategia en programas universitarios de administración en Medellín, Colombia. http://repository.ucc.edu.co/handle/ucc/274

Navas Rios, M., \& Romero-González, Z. (2016). Responsabilidad social universitaria. Saber, Ciencia Y Libertad, 11(1), 187-196. https://doi.org/10.18041/2382-3240/saber.2016v11n1.503

Ovans, Andrea, (2015). ¿What Is Strategy, Again?. Harvard Business Review, USA https://hbr. org/2015/05/what-is-strategy-again

Perdomo-Ortiz, Jesús. (2003). La arquitectura organizacional y las capacidades estratégicas empresariales empresariales*. Innovar, 13(22), 55-62. http://www.scielo.org.co/scielo.php?script=sci_arttext\&pid=S0121-50512003000200005\&lng=en\&tlng=es

Pérez-Vallejo, Lidia María, Vilariño-Corella, Carlos Manuel, \& Ronda-Pupo, Guillermo Armando. (2017). El cambio organizacional como herramienta para coadyuvar con la implementación de la estrategia. Ingeniería Industrial, 38(3), 323-332. http://scielo.sld.cu/scielo.php?script=sci_arttext\&pid=S1815-59362017000300010\&lng=es\&tlng=es

Porter, Michael E., (2008). Las Cinco Fuerzas Competitivas que le dan forma a la estrategia. Harvard Business Review América Latina, Enero 2008, USA.

Porter, Michael E., (2008). ¿Qué es la Estrategia? Harvard Business Review América Latina, Noviembre 2008, USA.

Rhodes, Mark, (23 de enero de 2011). Strategy First... Then Structure. https://managementhelp.org/ blogs/strategicplanning/2011/01/23/194 
Suárez, Jesús; Ibarra, Santiago, (2002). La Teoría de los Recursos y las Capacidades. Un Enfoque Actual en la Estrategia Empresarial. Anales de estudios económicos y empresariales, ISSN 0213$7569, \mathrm{~N}^{\circ} 15,2002$, págs. 63-89, Universidad de Valladolid, España. 